\section{ECCOMAS}

\author{
Proceedia
}

UNCECOMP 2017

$2^{\text {nd }}$ ECCOMAS Thematic Conference on

Uncertainty Quantification in Computational Sciences and Engineering M. Papadrakakis, V. Papadopoulos, G. Stefanou (eds.)

Rhodes Island, Greece, 15-17 June 2017

\title{
ASSESSING HOW THE DEPENDENCE STRUCTURE AFFECTS THE RELIABILITY PARAMETER OF THE STRENGTH-STRESS MODEL
}

\author{
A. Barbiero \\ ${ }^{1}$ Department of Economics, Management and Quantitative Methods, Università degli Studi di Milano, \\ via Conservatorio 7, 20122 Milan (Italy) \\ e-mail: alessandro.barbiero@unimi.it
}

Keywords: Bivariate random variable, Copula, Farlie-Gumbel-Morgenstern distributions, Fréchet bounds, Statistical dependence

\begin{abstract}
The stress-strength model is a basic modeling tool in reliability analysis. In simple terms, it considers a component (or a system) with an intrinsic strength $Y$, which is subjected to a stress $X$; the component works if and only if $Y$ is greater than $X$. If stress and strength are regarded as random variables, then the probability that the component works is given by $P(X<Y)$ and is usually called "reliability parameter". Statistical independence is usually assumed between the two random variables $X$ and $Y:$ for this case, the literature on this topic is particularly rich. This strong assumption, in fact, makes the calculation and estimation of the reliability parameter $R$ more tractable. However, this hypothesis is not always verified in practice, and this translates into an over- or under-estimation of $R$. To avoid this drawback, statistical dependence can be introduced and modeled between $X$ and $Y$, for example resorting to copulas. In some recent works, the problem of computing and estimating $R$ is considered when the stress and strength variables, belonging to the same parametric family of distributions, are linked by a specific copula. In this work, we further consider the computational issues related to this copula approach applied to the stress-strength model, when other families of copulas are selected. A sort of sensitivity analysis is performed in order to assess how the value of the reliability parameter is affected by the choice of the copula binding $X$ and $Y$ together and of its parameters. As a limit case, we consider the situation where no information on the dependence structure of the stress and strength margins is available and try to provide lower and upper bounds for $R$.
\end{abstract}




\section{INTRODUCTION}

The stress-strength model is a basic modeling tool in reliability analysis. In simple terms, it considers a component (or a system) with an intrinsic strength $Y$, which is subjected to a stress $X$; the component works if and only if $Y$ is greater than $X$; if stress and strength are regarded as random variables (r.v.s), then the probability that the component works is given by $P(X<Y)$ and is usually called "reliability parameter". The stress-strength model has many applications, especially in engineering concepts such as structures, deterioration of rocket motors, static fatigue of ceramic components, fatigue failure of aircraft structures, and the aging of concrete pressure vessels [1].

Statistical independence is usually assumed between the two r.v.s $X$ and $Y$ : for this case, the literature on this topic is particularly rich. The algebraic form for $R$ has been worked out for the majority of the well-known distributions, including normal, uniform, exponential, gamma, beta, extreme value, Weibull, Laplace, logistic, and Pareto distributions [2]. This strong assumption, in fact, makes the calculation and estimation of the reliability parameter $R$ more tractable. However, this hypothesis is not always verified in practice, and this translates into an overor under-estimation of $R$. To avoid this drawback, statistical dependence can be introduced and modeled between $X$ and $Y$, for example resorting to copulas. Citing [3], "the use of a joint distribution for stress and strength in estimating $R$ is justified by the practice of using stronger prototypes in worse environments which cause greater stresses. Moreover, the use of two independent random samples- one on $X$ and the other on $Y$-to estimate $R$ lacks support from pragmatic considerations".

The case when $(X, Y)$ follows a bivariate normal distribution has been investigated by [3, $4,5]$. [1] and [6] considered the problem of computing and estimating $R$ for some bivariate gamma and exponential distributions, respectively. Within the Bayesian framework, reference [7] studied the estimation of the reliability parameter assuming for the stress and strength variables asymmetric distributions obtained by skewing scale mixtures of normals; the margins are linked by the Gaussian copula. In [8] a stress-strength model is investigated with stress and strength marginally distributed as non-identical Dagum r.v.s and their dependence described by a Frank copula. In [9] the problem of estimation of the reliability parameter is considered when the Farlie-Gumbel-Morgenstern copula is used to link stress and strength variables, whose marginal distributions both belong to the Burr system. More recently, in [10], an ampler study on the effect of statistical dependence on the distribution of the functions of r.v.s deals also with the computation of $R$ when several statistical distributions are chosen for both $X$ and $Y$ and for various copula structures.

In this work, we further consider the computational issues related to this copula approach applied to the stress-strength model, when other families of copulas are selected. The rest of the paper is organized as follows. In Section 2, we recall some basic notions about copulas. In Section 3, after recalling the main results about the computation of the reliability parameter for stress-strength models involving copulas, we first derive its explicit expression when both stress and strength belong to the exponential family and other copula families than those already analyzed in the literature model their dependence. Then, in Section 4, we consider the situation where no information on the dependence structure of the stress and strength margins is available and try to provide lower and upper bounds for $R$. We examine the case of normally distributed and Cauchy distributed stress and strength. Section 5 concludes the paper with some possible research perspectives. 


\section{COPULAS}

Copulas allow to separate the study of the marginal distributions of a multivariate model from the study of the dependence structure and then to combine more marginal distributions with a variety of possible dependence structures. Formally, a $d$-dimensional copula $C\left(u_{1}, \ldots, u_{d}\right)$ is simply a joint cumulative distribution function (c.d.f.) in $[0,1]^{d}$ with standard uniform marginal c.d.f.s. The importance of copulas in the study of multivariate c.d.f.s is summarized by the theorem of Sklar [11, 12], which we briefly reprise here.

Let $F$ be a joint distribution function with margins $F_{1}, \ldots, F_{d}$. Then there exists a copula $C:[0,1]^{d} \rightarrow[0,1]$ such that, for all $x_{1}, \ldots, x_{d}$ in $\overline{\mathbb{R}}$,

$$
F\left(x_{1}, \ldots, x_{d}\right)=C\left(F_{1}\left(x_{1}\right), \ldots, F_{d}\left(x_{d}\right)\right) .
$$

If the margins are continuous, then $C$ is unique and is given by

$$
C\left(u_{1}, \ldots, u_{d}\right)=F\left(F_{1}^{-1}\left(u_{1}\right), \ldots, F_{d}^{-1}\left(u_{d}\right)\right),
$$

otherwise $C$ is uniquely determined on $\operatorname{Ran}\left(F_{1}\right) \times \cdots \times \boldsymbol{\operatorname { R a n }}\left(F_{d}\right)$, with $\boldsymbol{\operatorname { R a n }}\left(F_{i}\right)$ denoting the range of $F_{i}$. Conversely, if $C$ is a copula and $F_{1}, \ldots, F_{d}$ are univariate c.d.f.s, then the function $F$ defined in (1) is a joint c.d.f. with margins $F_{1}, \ldots, F_{d}$.

Since in the following we are interested in modeling the dependence of stress and strength variables in a standard stress-strength model, we can limit our focus to two r.v.s, $X \sim F$ and $Y \sim G$, and a bivariate copula $(d=2)$, denoted by $C(u, v)$. There is a large number of parametric families of copulas. Among them, we recall the elliptical copulas, comprising the well-known Gaussian copula, which is the (unique) copula that can be "extracted" by a bivariate normal distribution and is characterized by the parameter $\rho$ (the correlation coefficient). This copula does not have an explicit expression, it can be written as:

$$
C_{\rho}^{G a}=\Phi_{\rho}\left(\Phi^{-1}(u), \Phi^{-1}(v)\right),
$$

where $\Phi^{-1}$ is the inverse of the c.d.f. of a standard normal and $\Phi_{\rho}$ the joint c.d.f. of a standard bivariate normal r.v. with correlation $\rho$. Another important class of copulas is the Archimedean family, which comprises, for example, Frank, Clayton, and Gumbel copulas. They are often employed since they usually possess an explicit expression and are characterized by a single parameter controlling dependence, also in higher dimensions. Particular and very important types of copulas are the independence copula, $\Pi(u, v)=u v$, the comonotonicity copula, $M(u, v)=\min \{u, v\}$, and the countermonotonicity copula $W(u, v)=\max \{u+v-1,0\}$. For any copula $C(u, v)$, the following inequality holds:

$$
W(u, v) \leq C(u, v) \leq M(u, v) .
$$

For continuous r.v.s, the comonotonicity copula is the copula of two perfectly positive-dependent r.v.s: in this case, $(X, Y) \stackrel{d}{=}\left(F^{-1}(U), G^{-1}(U)\right)$; the countermonotonicity copula is the copula of two perfectly negative-dependent r.v.s.: in this case, $(X, Y) \stackrel{d}{=}\left(F^{-1}(U), G^{-1}(1-U)\right)$; the independence copula is the copula of two independent r.v.s. Note that the Gaussian copula (3) comprises the comonotonicity, countermonotonicity, and the independence copulas as limiting cases (for $\rho \rightarrow+1, \rho \rightarrow-1$, and $\rho=0$, respectively). 


\section{RELIABILITY FOR STRESS-STRENGTH MODEL WITH DEPENDENT VARI- ABLES}

If the continuous stress and strength r.v.s $X \sim F$ and $Y \sim G$ are linked by a copula $C$, then the stress-strength parameter $R$ is given by (see [10])

$$
P(X-Y<0)=1-\int_{0}^{1} \dot{C}_{1}\left(1-w, G\left(F^{-1}(1-w)\right)\right) \mathrm{d} w
$$

being $\dot{C}_{1}=\partial C(u, v) / \partial u=P(V \leq v \mid U=u)$. If additionally $X$ and $Y$ are both positive r.v.s, then the stress-strength parameter $R$ is given also by (see [10] again)

$$
P\left(\frac{X}{Y}<1\right)=1-\int_{0}^{1} \dot{C}_{1}\left(w, G\left(F^{-1}(w)\right)\right) \mathrm{d} w .
$$

Exploiting formulas (5) or (6), in [10] the expressions of the reliability parameter have been explicitly or numerically found for some possible choices of $F$ and $G$ (namely, uniform and exponential) and the linking copulas (Farlie-Gumbel-Morgenstern, Frank, Clayton and Gumbel).

In the next two subsections, we first examine two other kind of copulas and compute the reliability parameter when stress and strength follow an exponential distribution; then we provide sharp bounds for the reliability parameter when no information is available about the copula linking normal or Cauchy stress and strength distributions.

\subsection{Extended Farlie-Gumbel-Morgenstern copula}

The Farlie-Gumbel-Morgenstern [13], henceforth FGM, is a well-known family of copulas allowing for a moderate level of dependence:

$$
C(u, v)=u v(1+\theta(1-u)(1-v)),-1 \leq \theta \leq+1 .
$$

It can be easily shown that for this copula $\rho_{u v}=\theta / 3$, and then $\rho_{u v}$ is bounded between $-1 / 3$ and $1 / 3$. [14] showed that if the margins linked by the FGM copula are absolutely continuous, Pearson's correlation cannot exceed $1 / 3$. Several extensions of this family have been proposed, especially for enlarging the range of possible correlation values.

Here we first consider the following extension, which is due to [15] and described also in [16]:

$$
C(u, v)=u v+\theta u^{b} v^{b}(1-u)^{a}(1-v)^{a}, a, b \geq 1 .
$$

We set $a=2, b=1$ in Eq.(8) (then the constraint on $\theta$ is $-1 \leq \theta \leq+3$ ). The conditional distribution $\dot{C}_{1}$ can be easily derived:

$$
\dot{C}_{1}=\frac{\partial C(u, v)}{\partial u}=v+\theta v(1-v)^{2}\left(1+3 u^{2}-4 u\right)
$$

If $X$ and $Y$ follow exponential distributions with rate parameters $\lambda_{x}$ and $\lambda_{y}$, respectively, linked by the above extended FGM copula, then, since $F^{-1}(w)=-\log (1-w) / \lambda_{x}$ and $G\left(F^{-1}(1-\right.$ 
$w))=1-w^{\lambda_{y}} / \lambda_{x}$, the reliability parameter provided by Eq.(5) takes the following form:

$$
\begin{aligned}
R & =1-\int_{0}^{1}\left(1-w^{\alpha}\right)+\theta\left(1-w^{\alpha}\right) w^{2 \alpha}\left[1+3(1-w)^{2}-4(1-w)\right] \mathrm{d} w \\
& =1-\int_{0}^{1}\left(1-w^{\alpha}\right)+\theta\left(3 w^{2 \alpha+2}-2 w^{2 \alpha+1}-3 w^{3 \alpha+2}+2 w^{3 \alpha+1}\right) \mathrm{d} w \\
& =1-\left[w-\frac{w^{\alpha+1}}{\alpha+1}+\theta\left(\frac{3 w^{2 \alpha+3}}{2 \alpha+3}-\frac{2 w^{2 \alpha+2}}{2 \alpha+2}-\frac{3 w^{3 \alpha+3}}{3 \alpha+3}+\frac{2 w^{3 \alpha+2}}{3 \alpha+2}\right)\right]_{0}^{1} \\
& =\frac{1}{\alpha+1}-\theta\left(\frac{3}{2 \alpha+3}+\frac{2}{3 \alpha+2}-\frac{2}{\alpha+1}\right) \\
& =\frac{1}{\alpha+1}-\theta \frac{\alpha(\alpha-1)}{(\alpha+1)(2 \alpha+3)(3 \alpha+2)}
\end{aligned}
$$

where $\alpha=\lambda_{y} / \lambda_{x}$. For $\alpha>1$, i.e., if the expected value of $Y$ is smaller than the expected value of $X, R$ is a decreasing linear function of $\theta$; for $\alpha<1, R$ is an increasing linear function of $\theta$. When $\alpha=1, R$ is constant and equal to 0.5 . The range allowed to $R$ is quite narrow; for example, when $\alpha=1 / 3, R$ goes from 0.7348485 to 0.7954545 . If $\theta=0$, Eq.(10) boils down to the usual formula for two independent exponential distributions: $R=\frac{1}{\alpha+1}=\frac{\lambda_{x}}{\lambda_{x}+\lambda_{y}}$.

We consider now the following alternative extension of the FGM copula (see again [15] and [16]):

$$
C(u, v)=u v+\theta u v\left(1-u^{p}\right)\left(1-v^{p}\right), p>0 .
$$

Let us set $p=2$ in Eq.(11) (then the constraint on $\theta$ is $-1 / 4 \leq \theta \leq 1 / 2$ ). The conditional distribution $\dot{C}_{1}$ becomes:

$$
\dot{C}_{1}=v+\theta v\left(1-v^{2}\right)\left(1-3 u^{2}\right)
$$

and then from Eq.(6) we derive:

$$
\begin{aligned}
R & =1-\int_{0}^{1}\left(1-w^{\alpha}\right)+\theta\left(1-w^{\alpha}\right)\left[1-\left(1-w^{\alpha}\right)^{2}\right]\left[1-3(1-w)^{2}\right] \mathrm{d} w \\
& =1-\int_{0}^{1}\left(1-w^{\alpha}\right)+\theta\left(-2 w^{3 \alpha}+6 w^{2 \alpha}-4 w^{\alpha}-3 w^{3 \alpha+2}+9 w^{2 \alpha+2}-6 w^{\alpha+2}\right. \\
& \left.+6 w^{3 \alpha+1}-18 w^{2 \alpha+1}+12 w^{\alpha+1}\right) \mathrm{d} w \\
& =1-\left[w-\frac{w^{\alpha}}{\alpha+1}+\theta\left(\frac{6 w^{2 \alpha+1}}{2 \alpha+1}+\frac{6 w^{3 \alpha+2}}{3 \alpha+2}+\frac{12 w^{\alpha+2}}{\alpha+2}+\frac{9 w^{2 \alpha+3}}{2 \alpha+3}+\right.\right. \\
& \left.\left.-\frac{2 w^{3 \alpha+1}}{3 \alpha+1}-\frac{6 w^{\alpha+3}}{\alpha+3}-\frac{14 w^{\alpha+1}}{\alpha+1}\right)\right]_{0}^{1} \\
& =\frac{1}{\alpha+1}-\theta\left(\frac{6}{2 \alpha+1}+\frac{6}{3 \alpha+2}+\frac{12}{\alpha+2}+\frac{9}{2 \alpha+3}-\frac{2}{3 \alpha+1}-\frac{6}{\alpha+3}-\frac{14}{\alpha+1}\right) \\
& =\frac{1}{\alpha+1}-\theta \frac{(\alpha-1) \alpha\left(30 \alpha^{4}+251 \alpha^{3}+470 \alpha^{2}+251 \alpha+30\right)}{(\alpha+1)(\alpha+2)(\alpha+3)(2 \alpha+1)(2 \alpha+3)(3 \alpha+1)(3 \alpha+2)}
\end{aligned}
$$

As for the previous case, for $\alpha>1, R$ is a decreasing linear function of $\theta$; for $\alpha<1, R$ is an increasing linear function of $\theta$. When $\alpha=1, R$ is constant and equal to 0.5 . The range allowed to $R$ is quite narrow; for example, when $\alpha=1 / 3, R$ goes from 0.7243506 to 0.8012987 . If $\theta=0$, Eq.(13) boils down to the usual formula for two independent exponential distributions. 


\subsection{Ali-Mikhail-Hak copula}

We consider the following family of Archimedean copulas, known as Ali-Mikhail-Hak family [17]:

$$
C(u, v)=\frac{u v}{1-\theta(1-u)(1-v)},-1 \leq \theta \leq+1 .
$$

For $\theta=0$, we obtain the independence copula, whereas positive (negative) values of $\theta$ lead to positive (negative) dependence. The conditional distribution $\dot{C}_{1}$ can be easily derived:

$$
\dot{C}_{1}=\frac{\partial C(u, v)}{\partial u}=\frac{v(1-\theta+\theta v)}{[1-\theta(1-u)(1-v)]^{2}} .
$$

If $X$ and $Y$ follow exponential distributions with parameters $\lambda_{x}$ and $\lambda_{y}$, respectively, linked by a copula belonging to the Ali-Mikhail-Hak family, then the reliability parameter is provided, after some calculations, by Eq.(5) or (6):

$$
R=1-\int_{0}^{1} \frac{\left(1-w^{\alpha}\right)\left(1-\theta w^{\alpha}\right)}{\left(1-\theta w^{\alpha+1}\right)^{2}} \mathrm{~d} w
$$

The integral is not (easily) analytically solvable except for integer values of $\alpha$; in particular, if $\alpha=1$, we get

$$
R=1-\left.\frac{2 x \theta-\theta-1}{2 \theta(1-\theta x)^{2}}\right|_{0} ^{1}=1-\left(-\frac{1}{2 \theta}-\frac{-\theta-1}{2 \theta}\right)=\frac{1}{2}
$$

for any possible value of $\theta \in[-1,+1]$, as one could expect. However, in general, the integral in (16) can be solved numerically through any common statistical or mathematical software, such as $\mathrm{R}$ or Mathematica, and then it is possible, for example, to plot the graph of $R$ as a function of $\theta$. Such graphs, for several choices of $\alpha$, are presented in Figure 1: note that $R$ is an increasing (decreasing) function of $\theta$ for $\alpha$ smaller (greater) than 1 . The range allowed to $R$ looks larger than for the extended FGM copulas seen before, for a fixed value of $\alpha$; for example, when $\alpha=1 / 3, R$ is allowed to vary between from 0.717419 to 0.8390486 .

\section{LOWER AND UPPER BOUNDS FOR $R$ WHEN NO INFORMATION IS AVAIL- ABLE ABOUT DEPENDENCE}

Suppose again that $X$ and $Y$ are r.v.s with c.d.f.s $F$ and $G$, respectively. Let $S$ denote the c.d.f. of the sum $X+Y$, i.e., $S(z)=P(X+Y<z)$. A classical problem in probability is finding $S^{\wedge}(z)=\sup S(z)$ and $S^{\vee}(z)=\inf S(z)$, where the supremum and infimum are taken over the Fréchet-Hoeffding class $H(F, G)$ of all joint c.d.f.s $H$ with marginals $F$ and $G$. This problem leads to copulas naturally, since, as we have seen in Section 2, if $H$ is the joint distribution function of $X$ and $Y$, then $H(x, y)=C(F(x), G(y))$ for at least one copula $C$ (exactly one if $X$ and $Y$ are continuous). The following result holds (see [12], p.228):

$$
\begin{array}{r}
S^{\vee}(z) \leq S(z) \leq S^{\wedge}(z) \\
S^{\vee}(z)=\sup _{x+y=z} W(F(x), G(y)) \\
S^{\wedge}(z)=\inf _{x+y=z} \tilde{W}(F(x), G(y))
\end{array}
$$

with $\tilde{W}=u+v-W(u, v)=\min \{u+v, 1\}$. 


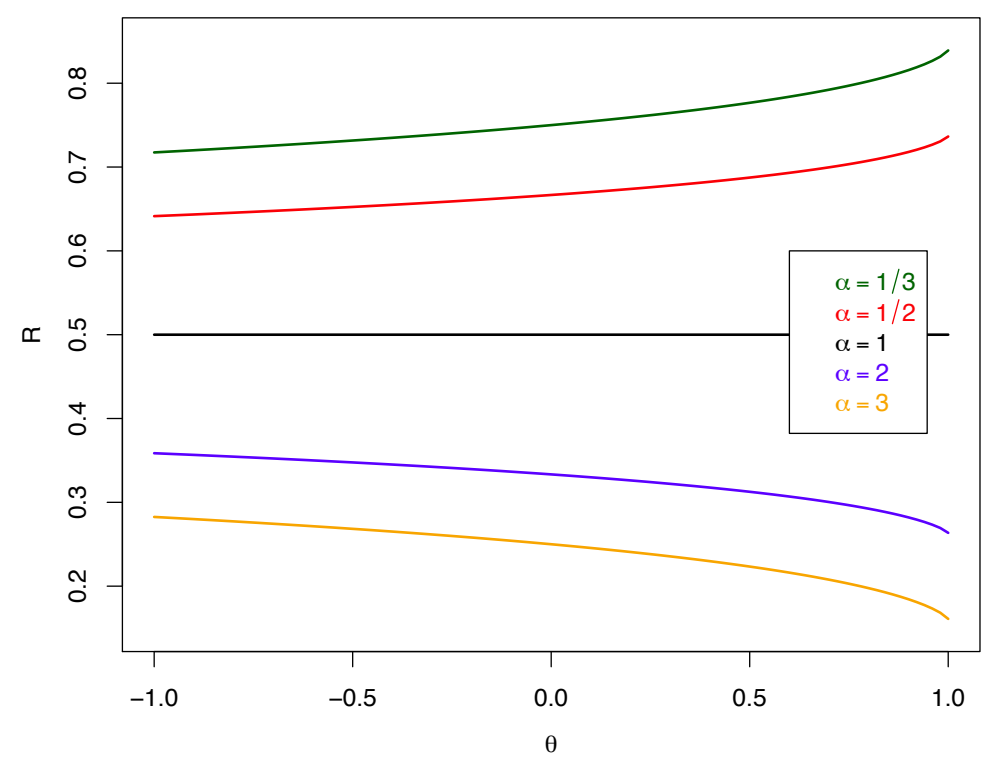

Figure 1: The reliability parameter $R$ for two exponential distributions (with $\alpha=\lambda_{y} / \lambda_{x}$ ) linked by Ali-MikhailHaq copula with parameter $\theta$

If instead of the $Y$ r.v. we consider its opposite $-Y$, with c.d.f. $1-G(-y)$, and let $z=0$, the problem above turns into finding the lower and upper bound (say, $R^{\vee}$ and $R^{\wedge}$ ) of the reliability parameter of a stress-strength model with stress $X \sim F$ and strength $Y \sim G$ over all joint c.d.f.s $H$ with marginals $F$ and $G: R^{\vee} \leq R=P(X<Y) \leq R^{\wedge}$. Adjusting the results reported in [12, p.229-231], we can state that the lower and upper bounds $R^{\vee}$ and $R^{\wedge}$ are sharp since they are attained by (at least) one copula each. Recalling that if the copula of $(X, Y)$ is $C(u, v)$, then the copula of $(X,-Y)$ is $u-C(u, 1-v)$, we derive for the lower bound:

$$
C^{\vee}(u, v)= \begin{cases}\min \left\{u-R^{\vee}, v\right\} & {\left[R^{\vee}, 1\right] \times\left[0,1-R^{\vee}\right]} \\ \max \{0, u+v-1\} & \text { elsewhere; }\end{cases}
$$

for the upper bound:

$$
C^{\wedge}(u, v)= \begin{cases}\min \left\{u, v-\left(1-R^{\wedge}\right)\right\} & {\left[0, R^{\wedge}\right] \times\left[1-R^{\wedge}, 1\right]} \\ \max \{0, u+v-1\} & \text { elsewhere }\end{cases}
$$

\subsection{Normal stress and strength}

Let us consider the case of normal stress and strength: $X \sim N\left(\mu_{x}, \sigma_{x}^{2}\right), Y \sim N\left(\mu_{y}, \sigma_{y}^{2}\right)$. If $\sigma_{x}^{2}=\sigma_{y}^{2}=\sigma^{2}$ it can be shown (properly adapting the results presented in [12, p.232-233]) that

$$
\begin{gathered}
R^{\vee}= \begin{cases}0 & \eta \leq 0 \\
2 \Phi\left(\frac{\eta}{2 \sigma}\right)-1 & \eta \geq 0\end{cases} \\
R^{\wedge}= \begin{cases}2 \Phi\left(\frac{\eta}{2 \sigma}\right) & \eta \leq 0 \\
1 & \eta \geq 0\end{cases}
\end{gathered}
$$


where $\eta=\mu_{y}-\mu_{x}$. If the variances are not the same,

$$
R^{\vee}=\Phi\left(\frac{-\sigma_{x} \eta-\sigma_{y} \phi}{\sigma_{y}^{2}-\sigma_{x}^{2}}\right)+\Phi\left(\frac{\sigma_{y} \eta-\sigma_{x} \phi}{\sigma_{y}^{2}-\sigma_{x}^{2}}\right)-1
$$

and

$$
R^{\wedge}=\Phi\left(\frac{-\sigma_{x} \eta+\sigma_{y} \phi}{\sigma_{y}^{2}-\sigma_{x}^{2}}\right)+\Phi\left(\frac{\sigma_{y} \eta+\sigma_{x} \phi}{\sigma_{y}^{2}-\sigma_{x}^{2}}\right),
$$

where $\phi=\left[\eta^{2}+2\left(\sigma_{y}^{2}-\sigma_{x}^{2}\right) \log \left(\sigma_{x} / \sigma_{x}\right)\right]^{1 / 2}$.

Let us remember that if $X$ and $Y$ are assumed to be independent normal r.v.s, then the reliability parameter $R$ would be equal to

$$
R=\Phi\left(\frac{\eta}{\sqrt{\sigma_{x}^{2}+\sigma_{y}^{2}}}\right) .
$$

If $X$ and $Y$ are comonotonic, then

$$
R=\Phi\left(\frac{\eta}{\left|\sigma_{y}-\sigma_{x}\right|}\right)
$$

in case of unequal variances; in case of equal variances, $R=1$ if $\eta>0 ; R=0$ if $\eta \leq 0$. If $X$ and $Y$ are countermonotonic, then it can be easily proved that

$$
R=\Phi\left(\frac{\eta}{\sigma_{y}+\sigma_{x}}\right)
$$

Example 1 (normal stress and strength with equal variances). Let $\mu_{x}=6, \mu_{y}=7$, $\sigma_{x}=\sigma_{y}=2$. If $X$ and $Y$ are assumed to be independent r.v.s, then the reliability parameter $R$, applying Eq.(25), would be equal to 0.6382. Lower and upper bounds for $R$ are provided through Eqs.(21) and (22) as $R^{\vee}=0.1974$ and $R^{\wedge}=1$. From Eq.(27), the value of $R$ corresponding to the countermonotonicity copula $W(u, v)$ is $R=0.5987$; for the comonotonicity copula $M(u, v)$, applying Eq.26, we have $R=1$.

Example 2 (normal stress and strength with unequal variances). Let $\mu_{x}=6, \sigma_{x}=1$, $\mu_{y}=7, \sigma_{y}=2$. If $X$ and $Y$ are assumed to be independent r.v.s, then, applying Eq.(25), the reliability parameter $R$ would be equal to 0.6726 . Lower and upper bounds for $R$ are provided through Eqs.(23) and (24) as $R^{\vee}=0.3451$ and $R^{\wedge}=0.9551$. From Eq.(27), the value of $R$ corresponding to the countermonotonicity copula is $R=0.6306$; for the comonotonicity copula (applying Eq.26), we have $R=0.8413$. Copulas leading to $R^{\vee}$ and $R^{\wedge}$, given by Eqs.(19) and (20), are graphically displayed in Figure 2.

\subsection{Cauchy stress and strength}

Let us now focus on the Cauchy distribution, whose density function is given by

$$
f(t ; \alpha, \beta)=\frac{1}{\pi \beta\left[1+\left(\frac{t-\alpha}{\beta}\right)^{2}\right]}, \quad x, \alpha \in \mathbb{R}, \beta \in \mathbb{R}^{+},
$$

and whose c.d.f. is

$$
F(t ; \alpha, \beta)=\frac{1}{2}+\frac{1}{\pi} \arctan \left(\frac{t-\alpha}{\beta}\right)
$$



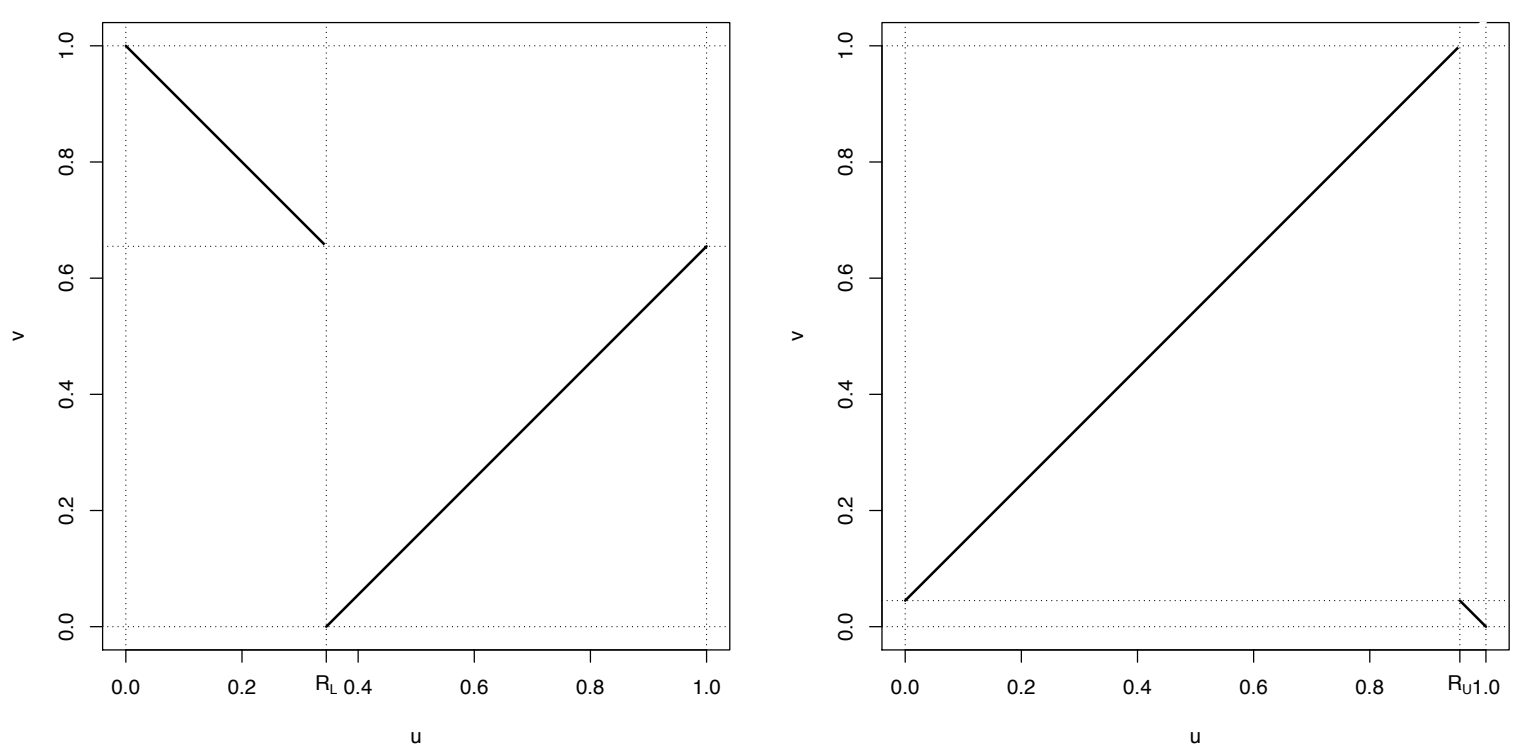

Figure 2: Reliability parameter for normal stress $X$ and strength $Y$. Representation of the supports of copulas leading to the lower bound $\left(C^{\vee}(u, v)\right.$, left $)$ and upper bound $\left(C^{\wedge}(u, v)\right.$, right) when $X \sim N\left(\mu_{x}=6, \sigma_{x}=1\right)$ and $Y \sim N\left(\mu_{y}=7, \sigma_{y}=2\right) . R_{L}$ stands for the lower bound $R^{\vee}$ and $R_{U}$ for the upper bound $R^{\wedge}$.

If we assume that $X$ and $Y$ are r.v.s with Cauchy distribution with location parameters $\alpha_{x}$ and $\alpha_{y}$, and scale parameters $\beta_{x}$ and $\beta_{y}$, respectively, then we have the following bounds for the reliability parameter $R$, derived rearranging [12, p.234]. If $\beta_{x}=\beta_{y}=\beta$,

$$
\begin{aligned}
& R^{\vee}= \begin{cases}0 & \eta \leq 0 \\
\frac{2}{\pi} \arctan \left(\frac{\eta}{2 \beta}\right) & \eta \geq 0\end{cases} \\
& R^{\wedge}= \begin{cases}1+\frac{2}{\pi} \arctan \left(\frac{\eta}{2 \beta}\right) & \eta \leq 0 \\
1 & \eta \geq 0\end{cases}
\end{aligned}
$$

where $\eta=\alpha_{y}-\alpha_{x}$. If $\beta_{x} \neq \beta_{y}$,

$$
\begin{aligned}
& R^{\vee}=\frac{1}{\pi}\left[\arctan \left(\frac{-\eta+\beta_{y} \phi}{\beta_{y}-\beta_{x}}\right)+\arctan \left(\frac{\eta-\beta_{x} \phi}{\beta_{y}-\beta_{x}}\right)\right] \\
& R^{\wedge}=1+\frac{1}{\pi}\left[\arctan \left(\frac{-\eta-\beta_{y} \phi}{\beta_{y}-\beta_{x}}\right)+\arctan \left(\frac{\eta+\beta_{x} \phi}{\beta_{y}-\beta_{x}}\right)\right]
\end{aligned}
$$

where $\phi=\left[\left(\eta^{2}+\left(\beta_{y}-\beta_{x}\right)^{2}\right) /\left(\beta_{x} \beta_{y}\right)\right]^{1 / 2}$.

Note that for independent r.v.s we have $R=\frac{1}{2}+\frac{1}{\pi} \arctan \left(\frac{\eta}{\beta_{x}+\beta_{y}}\right)$. If the copula linking $X$ and $Y$ is the comonotonicity copula we have $R=\frac{1}{2}+\frac{1}{\pi} \arctan \left(\frac{\eta}{\left|\beta_{y}-\beta_{x}\right|}\right)$, if $\beta_{x} \neq \beta_{y}$. Otherwise, if $\beta_{x}=\beta_{y}, R=1$ if $\eta>0, R=0$ if $\eta \leq 0$. If the copula linking $X$ and $Y$ is the countermonotonicity copula we have $R=\frac{1}{2}+\frac{1}{\pi} \arctan \left(\frac{\eta}{\beta_{x}+\beta_{y}}\right)$, as for the independence copula. Note then how two different copulas linking $X$ and $Y$ provide in this case the same value for the probability $P(X<Y)$. 
Example 3 (Cauchy stress and strength equal scale parameters). Let $\alpha_{x}=6, \alpha_{y}=7$, $\beta_{x}=\beta_{y}=2$. Lower and upper bounds for $R$ are provided as $R^{\vee}=0.1560$ and $R^{\wedge}=1$. Note that if $X$ and $Y$ were independent, then $R=0.5780$ and the same result would hold if they were countermonotonic; if $X$ and $Y$ were comonotonic, $R=1$.

Example 4 (Cauchy stress and strength unequal scale parameters). Let $\alpha_{x}=6, \beta_{x}=1$, $\alpha_{y}=7, \beta_{y}=2$. Lower and upper bounds for $R$ are provided as $R^{\vee}=0.25$ and $R^{\wedge}=0.9548$. Note that if $X$ and $Y$ were independent, then $R=0.6024$, and the same result would hold if they were countermonotonic; if $X$ and $Y$ were comonotonic, $R=0.75$.

\section{CONCLUSIONS}

In this work, we considered the problem of computing the reliability of the stress-strength model when stress and strength variables are dependent. We first examined the case of exponential variables linked by three different types of copulas, deriving analytical expression for the reliability parameter and examining its relationship with the copula parameter. Then, focusing on normal or Cauchy distributed stress and strength, we derived lower and upper bound for the reliability parameter when no assumption is made on their copula.

As for research perspectives, other distributions for stress and strength and other copula families can be examined and the corresponding value of $R$, or sharp lower and upper bounds for $R$, can be analytically or numerically derived. Generalizations to more complex stress-strength models, where stress and strength are themselves functions of several random components (i.e., $X=\xi\left(X_{1}, X_{2}, \ldots, X_{h}\right)$ and $Y=v\left(Y_{1}, Y_{2}, \ldots, Y_{k}\right)$, for some functions $\xi$ and $\left.v\right)$ will be considered. Another aspect to be developed is the estimation of lower and upper bounds for $R$ when only random samples from $X$ and $Y$ are available: this means that besides the uncertainty about the dependence structure (copula) of $X$ and $Y$, we will have to take into account also the uncertainty about (the parameters of) the margins, which have to be estimated from the samples.

\section{REFERENCES}

[1] S. Nadarajah, Reliability for some bivariate gamma distributions. Mathematical Problems in Engineering, 2005(2), 151-163, 2005.

[2] S. Kotz, Y. Lumelskii, M. Pensky, The stress-strength model and its generalizations. Theory and Applications. World Scientific, 2003.

[3] S.P. Mukherjee, L.K. Sharan, Estimation of failure probability from a bivariate normal stress-strength distribution, Microelectronics Reliability, 25, 699-702, 1985.

[4] D. Roy, Estimation of failure probability under a bivariate normal stress-strength distribution, Microelectronics Reliability, 33(15), 2285-2287, 1993.

[5] A. Barbiero, Interval estimators for reliability: the bivariate normal case, Journal of Applied Statistics, 39(3), 501-512, 2012.

[6] S. Nadarajah, Reliability for some bivariate exponential distributions. Mathematical Problems in Engineering, 1-14, 2006.

[7] F.J. Rubio, M.F. Steel, Bayesian Inference for $P(X<Y)$ Using Asymmetric Dependent Distributions, Bayesian Analysis, 8(1), 43-62, 2013. 
[8] F. Domma, S. Giordano, A stress-strength model with dependent variables to measure household financial fragility, Statistical Methods \& Applications, 21(3), 375-389, 2012.

[9] F. Domma, S. Giordano, A copula-based approach to account for dependence in stressstrength models, Statistical Papers, 54(3), 807-826, 2013.

[10] A. Dolati, R. Roozegar, N. Ahmadi, Z. Shishebor, The effect of dependence on distribution of the functions of random variables, Communications in Statistics-Theory and Methods, 2016 (to be published).

[11] A. Sklar, Fonctions de répartition à n dimensions et leurs marges, Publications de l'Institut de Statistique de l'Université de Paris, 8, 229-231, 1959.

[12] R.B. Nelsen, An Introduction to Copulas. Springer, 1999.

[13] D.J.G. Farlie, The performance of some correlation coefficient for a general bivariate distribution, Biometrika, 47, 307-323, 1960.

[14] W. Schucany, W.C. Parr, J.E. Boyer, Correlation structure in Farlie-Gumbel-Morgenstern distributions, Biometrika, 65(3), 650-653, 1978.

[15] C.D. Lai, M. Xie, A new family of positive quadrant dependent bivariate distributions, Statistics and Probability Letters, 46, 359-364, 2000.

[16] J. S. Huang, S. Kotz, Modifications of the Farlie-Gumbel-Morgenstern distributions. A tough hill to climb, Metrika, 49: 135145, 1999.

[17] M.M. Ali, N.N. Mikhail, M.S. Haq, A class of bivariate distributions including the bivariate logistic, Journal of Multivariate Analysis, 8(3): 405-412, 1978. 